\title{
On the Emergent Constraints of Climate Sensitivity
}

\author{
Xin Qu, Alex Hall, And Anthony M. DeAngelis \\ Department of Atmospheric and Oceanic Sciences, University of California, Los Angeles, Los Angeles, California
}

MARK D. ZelinKa AND STEPHEN A. KLEIN

Cloud Processes Research Group, Lawrence Livermore National Laboratory, Livermore, California

Hui Su, BAIJUn TIAN, AND CHENGXING ZHAI

Jet Propulsion Laboratory, California Institute of Technology, Pasadena, California

(Manuscript received 19 July 2017, in final form 25 October 2017)

\begin{abstract}
Differences among climate models in equilibrium climate sensitivity (ECS; the equilibrium surface temperature response to a doubling of atmospheric $\mathrm{CO}_{2}$ ) remain a significant barrier to the accurate assessment of societally important impacts of climate change. Relationships between ECS and observable metrics of the current climate in model ensembles, so-called emergent constraints, have been used to constrain ECS. Here a statistical method (including a backward selection process) is employed to achieve a better statistical understanding of the connections between four recently proposed emergent constraint metrics and individual feedbacks influencing ECS. The relationship between each metric and ECS is largely attributable to a statistical connection with shortwave low cloud feedback, the leading cause of intermodel ECS spread. This result bolsters confidence in some of the metrics, which had assumed such a connection in the first place. Additional analysis is conducted with a few thousand artificial metrics that are randomly generated but are well correlated with ECS. The relationships between the contrived metrics and ECS can also be linked statistically to shortwave cloud feedback. Thus, any proposed or forthcoming ECS constraint based on the current generation of climate models should be viewed as a potential constraint on shortwave cloud feedback, and physical links with that feedback should be investigated to verify that the constraint is real. In addition, any proposed ECS constraint should not be taken at face value since other factors influencing ECS besides shortwave cloud feedback could be systematically biased in the models.
\end{abstract}

\section{Introduction}

The equilibrium climate sensitivity (ECS), defined as the equilibrium global and annual-mean surface air temperature response to a doubling of atmospheric $\mathrm{CO}_{2}$ in general circulation models (GCMs), has consistently exhibited a large spread (ranging from 2 to $5 \mathrm{~K}$ ) since the first assessment of the Intergovernmental Panel for Climate Change in the 1990s (Mitchell et al. 1990; Kattenberg et al. 1996; Cubasch et al. 2001; Meehl et al. 2007; Collins et al. 2013). The spread in warming leads to spread in many warming-induced changes, such as changes in atmospheric circulation and the hydrological cycle (Lu et al. 2007; DeAngelis et al. 2015). Thus, it

Corresponding author: Xin Qu, xinqu@atmos.ucla.edu remains a significant barrier to the accurate assessment of societally important impacts of climate change.

Among all factors influencing ECS, shortwave (SW) cloud feedback (especially, the feedback associated with tropical low clouds) has long been recognized as the leading source of spread (Bony and Dufresne 2005; Andrews et al. 2012; Vial et al. 2013; Caldwell et al. 2016). This prompted numerous efforts to constrain the various components of SW cloud feedback (e.g., Clement et al. 2009; Bretherton 2015; Gordon and Klein 2014; Qu et al. 2014, 2015; Zhou et al. 2015; Ceppi et al. 2016; Tan et al. 2016). A method commonly used in these studies is the so-called emergent constraint approach. Emergent constraints associated with a particular feedback are strong statistical relationships between the feedback and observable characteristics of the current 
(a)

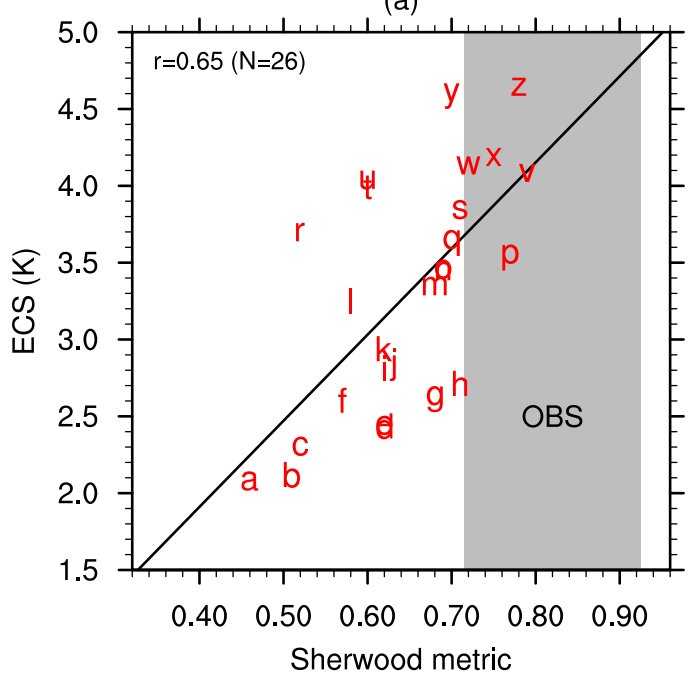

(c)

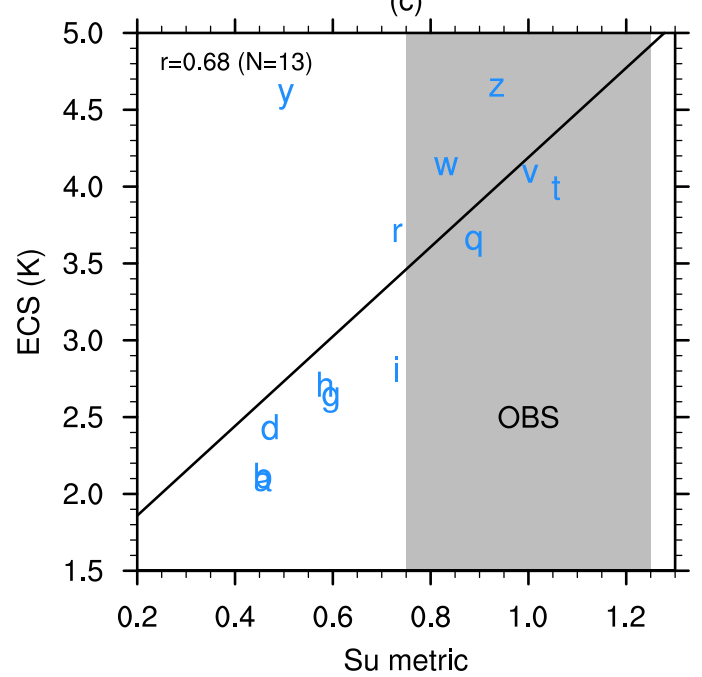

(b)

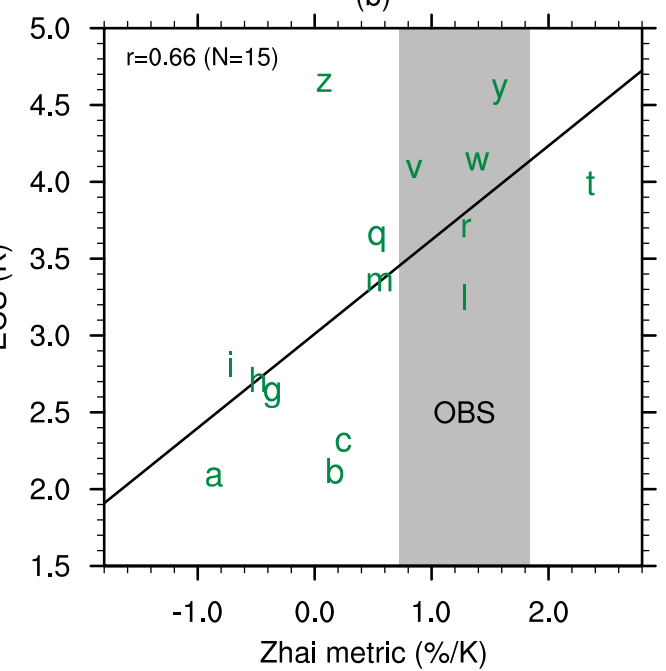

(d)

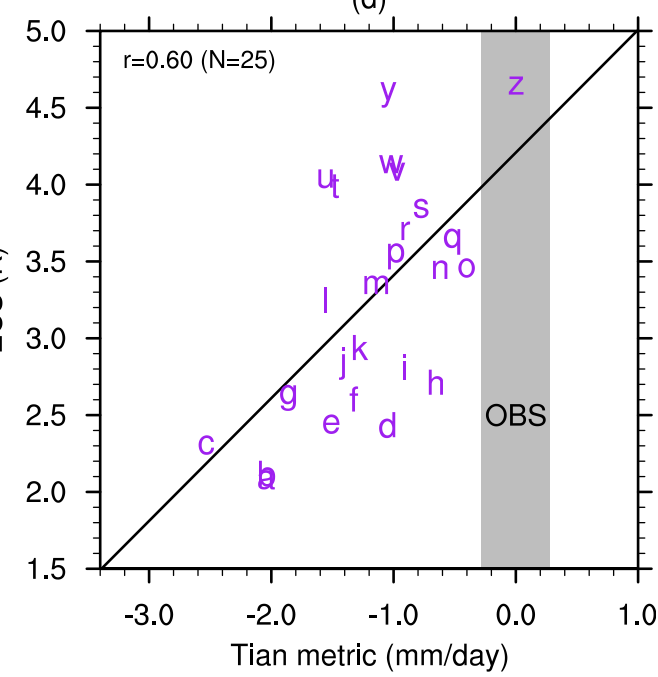

FIG. 1. Scatterplot of the ECS against four recently proposed metrics in a suite of CMIP5 GCMs (letters represent the GCMs from Table 1): (a) the Sherwood metric (Sherwood et al. 2014), (b) the Zhai metric (Zhai et al. 2015), (c) the Su metric (Su et al. 2014), and (d) the Tian metric (Tian 2015). In each diagram, the best-fit regression line is represented by the solid line, and the observational range of each metric is represented by the shaded area. The correlation $r$ between each metric and ECS and the number $N$ of GCMs involved are also shown at top left. To obtain positively signed correlations for all metrics, the signs of the Zhai and Tian metrics were reversed.

climate that emerge in collections of climate simulations and have a strong physical basis (Klein and Hall 2015).

In recent years, the emergent constraint approach has also been explored to directly constrain ECS (e.g., Fasullo and Trenberth 2012; Sherwood et al. 2014; Su et al. 2014; Tian 2015; Zhai et al. 2015; Brient and Schneider 2016), despite the fact that ECS is shaped by a number of feedback processes. Figure 1 depicts four recently proposed emergent constraints for ECS, each of which is associated with an observable metric of the current climate (Sherwood et al. 2014; Su et al. 2014; Tian 2015; Zhai et al. 2015). (See section 2 for the definition of these metrics.) All four metrics are reasonably well correlated with ECS, and comparison with their observational estimates suggests that ECS values in the upper half of the model range are more likely.

There are two fundamental questions surrounding the constraints on ECS. First, do all of them have a clear physical basis? Second, to what extent are they different from the direct emergent constraints of SW cloud feedback constructed previously (Clement et al. 2009; Bretherton 2015; Gordon and Klein 2014; Qu et al. 2014, 2015; Zhou et al. 2015; Ceppi et al. 2016)? A key step toward answering these questions is to figure out what 
drives the relationships between the emergent constraints and ECS (e.g., shown in Fig. 1). Given the importance of SW cloud feedback to ECS spread, it has been hypothesized that the correlations between some of the recently proposed metrics and ECS stem from a physical connection between the metrics and SW cloud feedback (Sherwood et al. 2014; Zhai et al. 2015; Brient and Schneider 2016). However, the validity of this hypothesis is not straightforward to assess because ECS is determined by multiple climate feedbacks and radiative forcing, which are generally not independent of one another and may combine in a nonlinear fashion (Caldwell et al. 2016).

In this study, we develop a statistical method to diagnose what drives the correlation between ECS and any given climatic metric $M$. In particular, a multivariate regression model, coupled with a backward selection process, is used to identify the feedbacks or forcing exhibiting a statistically significant relationship with $M$. This model is then used to decompose the correlation between ECS and $M$ into the contributions from each of the feedback and forcing terms. We apply this method to the four recently proposed metrics shown in Fig. 1 and to a suite of randomly generated artificial metrics that are constructed to be well correlated with ECS. The artificial metrics are a useful addition to our analysis because they allow us to examine whether the statistical properties of the four metrics in Fig. 1 are applicable to any other emergent constraints of ECS that have been proposed or may be proposed in the future (e.g., Fasullo and Trenberth 2012; Brient and Schneider 2016). An underlying assumption is that given the sufficiently large sample size of the artificial metrics, all possible emergent constraints are just a subset of the artificial metrics. This assumption is reasonable because a significant correlation with ECS is a prerequisite for any emergent constraint of ECS. We also hope that the inclusion of the artificial metrics will compensate for our exclusion of several other emergent constraints (e.g., Fasullo and Trenberth 2012; Brient and Schneider 2016).

\section{Data and methodology}

\section{a. Radiative forcing, climate feedbacks, and ECS}

Relying on values computed in a previous study (Caldwell et al. 2016), radiative forcing, climate feedbacks, and climate sensitivity in 26 GCMs participating in phase 5 of the Coupled Model Intercomparison Project (CMIP5; Table 1) are diagnosed via the Gregory method (Gregory et al. 2004). The main procedures to obtain them are described below [see Caldwell et al. (2016) for details]. First, surface air temperature $T$ and
TABLE 1. The 26 CMIP5 GCMs used in this study, their ECS values, and the metrics constructed in Sherwood et al. (2014), Zhai et al. (2015), Su et al. (2014), and Tian (2015). GCMs are organized in order of increasing ECS values. The number of the GCMs available varies with the metrics. Note that while only 15 GCMs have the Zhai metric available and 13 GCMs have the Su metric available, these GCMs cover the whole ECS range nicely. GCMs with the data available for APRP calculations are marked with asterisks in column 2. Note also that the Zhai metric was computed using a slightly modified methodology from the one described in Zhai et al. (2015) (see section 2). For convenience, the signs of the Zhai and Tian metrics were reversed. (Acronym expansions are available online at http://www.ametsoc.org/PubsAcronymList.)

\begin{tabular}{llccccc}
\hline \hline Letter & \multicolumn{1}{c}{ Name } & ECS & Sherwood & Zhai & Su & Tian \\
\hline $\mathrm{a}$ & INM-CM4.0* & 2.08 & 0.46 & -0.86 & 0.46 & -2.04 \\
$\mathrm{~b}$ & GISS-E2-R* & 2.12 & 0.51 & 0.18 & 0.46 & -2.03 \\
$\mathrm{c}$ & GISS-E2-H* & 2.31 & 0.52 & 0.25 & - & -2.53 \\
$\mathrm{~d}$ & GFDL-ESM2G* & 2.43 & 0.62 & - & 0.47 & -1.05 \\
$\mathrm{e}$ & GFDL-ESM2M* & 2.44 & 0.62 & - & - & -1.51 \\
$\mathrm{f}$ & IPSL-CM5B-LR* & 2.61 & 0.57 & - & - & -1.33 \\
$\mathrm{~g}$ & MRI-CGCM3* & 2.61 & 0.68 & -0.37 & 0.60 & -1.86 \\
$\mathrm{~h}$ & MIROC5* & 2.71 & 0.71 & -0.48 & 0.58 & -0.65 \\
$\mathrm{i}$ & NorESM1-M* & 2.81 & 0.62 & -0.72 & 0.73 & -0.91 \\
$\mathrm{j}$ & BCC_CSM1.1* & 2.84 & 0.63 & - & - & -1.42 \\
$\mathrm{k}$ & CCSM4* & 2.94 & 0.62 & - & - & -1.27 \\
$\mathrm{l}$ & CNRM-CM5* & 3.25 & 0.58 & 1.28 & - & -1.55 \\
$\mathrm{~m}$ & FGOALS-g2 & 3.35 & 0.68 & 0.55 & - & -1.14 \\
$\mathrm{n}$ & MPI-ESM-MR* & 3.45 & 0.69 & - & - & -0.62 \\
$\mathrm{o}$ & MPI-ESM-P* & 3.46 & 0.69 & - & - & -0.40 \\
$\mathrm{p}$ & ACCESS1.3* & 3.53 & 0.77 & - & - & -0.98 \\
$\mathrm{q}$ & MPI-ESM-LR* & 3.63 & 0.70 & 0.53 & 0.89 & -0.52 \\
$\mathrm{r}$ & CanESM2* & 3.70 & 0.52 & 1.30 & 0.73 & -0.90 \\
$\mathrm{~s}$ & ACCESS1.0* & 3.84 & 0.71 & - & - & -0.77 \\
$\mathrm{t}$ & GFDLCM3* & 3.99 & 0.60 & 2.36 & 1.06 & -1.48 \\
$\mathrm{u}$ & BNU-ESM* & 4.04 & 0.60 & - & - & -1.56 \\
$\mathrm{v}$ & CSIRO Mk3.6.0 & 4.08 & 0.79 & 0.85 & 1.00 & -0.97 \\
$\mathrm{w}$ & IPSL-CM5A-LR* & 4.13 & 0.72 & 1.39 & 0.83 & -1.02 \\
$\mathrm{x}$ & FGOALS-s2* & 4.19 & 0.75 & - & - & - \\
$\mathrm{y}$ & HadGEM2-ES* & 4.58 & 0.70 & 1.58 & 0.50 & -1.04 \\
$\mathrm{z}$ & MIROC-ESM* & 4.64 & 0.78 & 0.08 & 0.94 & 0.00 \\
\hline & & & & & &
\end{tabular}

radiative fluxes at the top of the atmosphere (TOA) are taken from the 150-yr CMIP5 abrupt4xCO2 simulations. In these simulations, atmospheric $\mathrm{CO}_{2}$ concentration is instantaneously quadrupled from its preindustrial level and then held constant. Then the climatological mean fields from the piControl experiments are subtracted from those of the abrupt $4 \mathrm{xCO} 2$ experiments, and Gregory-style regression analysis (Gregory et al. 2004) is done to compute radiative forcing $F$ as the intercept at $\Delta T=0$ and net feedback $\lambda$ as the regression slope (Table 2). Finally, ECS is computed as ECS $=-F / \lambda$ (Table 1$)$. Note that $F$ is halved in this calculation to represent the radiative forcing corresponding to a doubling of $\mathrm{CO}_{2}$. While a recent study suggests that the ECS diagnosed via the Gregory method may be a systematic underestimate (Andrews et al. 2015), this bias is generally small. More importantly, much of the intermodel spread 
TABLE 2. The ensemble-mean and standard deviation (in parentheses) of radiative forcing $\left(\mathrm{W} \mathrm{m}^{-2}\right)$ and climate feedbacks $\left(\mathrm{W} \mathrm{m}^{-2} \mathrm{~K}^{-1}\right.$ ) in 26 CMIP5 GCMs (Table 1 ). The feedbacks include $\lambda, \lambda_{\mathrm{Pl}}, \lambda_{\mathrm{WV}}, \lambda_{\mathrm{LR}}, \lambda_{\mathrm{SWcld}}, \lambda_{\mathrm{LWcld}}$, and $\lambda_{\mathrm{Alb}}$. The residual term of the kernel calculations (referred to as $\lambda_{\mathrm{Re}}$; see the text) is also shown. Note that the Pl, WV, and LR feedbacks are computed based on the Held and Shell (2012) definition of these feedbacks.

\begin{tabular}{lr}
\hline \hline Forcing/feedback & \multicolumn{1}{c}{ Values } \\
\hline$F$ & $3.41( \pm 0.53)$ \\
$\lambda$ & $-1.09( \pm 0.30)$ \\
$\lambda_{\mathrm{Pl}}$ & $-1.85( \pm 0.03)$ \\
$\lambda_{\mathrm{WV}}$ & $0.02( \pm 0.05)$ \\
$\lambda_{\mathrm{LR}}$ & $-0.15( \pm 0.11)$ \\
$\lambda_{\mathrm{SWcld}}$ & $-0.01( \pm 0.38)$ \\
$\lambda_{\mathrm{LWcld}}$ & $0.41( \pm 0.19)$ \\
$\lambda_{\mathrm{Alb}}$ & $0.38( \pm 0.09)$ \\
$\lambda_{\mathrm{Re}}$ & $0.11( \pm 0.13)$ \\
\hline
\end{tabular}

and variations in the true ECS are still captured by the Gregory method (Andrews et al. 2015).

Caldwell et al. (2016) use radiative kernels developed by Soden et al. (2008, hereafter Soden08) and Shell et al. (2008, hereafter Shell08) to break down $\lambda$ into individual feedbacks, including the Planck $(\mathrm{Pl}) \lambda_{\mathrm{Pl}}$, water vapor (WV) $\lambda_{\mathrm{WV}}$, lapse rate (LR) $\lambda_{\mathrm{LR}}, \mathrm{SW}$ cloud $\lambda_{\mathrm{SWcld}}$, longwave (LW) cloud $\lambda_{\text {LWcld }}$, and surface albedo $\lambda_{\text {Alb }}$ feedbacks (Table 2). The linearity assumed in the kernel calculations is not always valid (Vial et al. 2013). The deviation from linearity can be quantified by the difference between the net feedback diagnosed directly from model fluxes and the sum of the various kernelcomputed feedbacks. This term is hereafter referred to as the residual feedback term $\lambda_{\mathrm{Re}}$.

For either the Soden 08 or Shell08 kernel, the Pl, WV, and LR feedbacks can be defined traditionally (Soden08 and Shell08) or via the approach in Held and Shell (2012). Held and Shell (2012) suggest partitioning the WV feedback into three components: 1) the net radiative effect of the specific humidity change associated with relative humidity $(\mathrm{RH})$ changes, 2 ) the net radiative effect of the specific humidity change at constant RH assuming vertically uniform atmospheric warming, and 3) the net radiative effect of the specific humidity change at constant RH with vertically nonuniform atmospheric warming. They further suggest redefining the WV feedback as the first component only and adding the second component to the Pl feedback and the third to the LR feedback. This may be more desirable than the traditional partitioning because it avoids the well-known anticorrelation between the WV and LR feedbacks. Applying both partition methods to the two kernels, we have in total four estimates of the $\mathrm{Pl}, \mathrm{WV}$, and LR feedbacks. However, we find that the results are insensitive to the choice of either partition method or kernel. So for clarity, we only present the results performed with the Soden08 kernel and the Held and Shell (2012) definition of the feedbacks.

Given the importance of SW cloud feedback for ECS spread, we further break the SW cloud feedback down into its two components, one associated with changes in cloud amount and the other with changes in cloud scattering and absorption, using the approximate partial radiative perturbation (APRP) method (Taylor et al. 2007). It is possible to break down the second component further (into cloud scattering and absorption feedbacks), but there is no need to do so because the cloud scattering feedback generally dominates. The sum of SW scattering and absorption components is very similar to the SW optical depth feedback. There is excellent agreement between the kernel- and APRPcomputed SW cloud feedbacks. The cross-model correlation between the two quantities averaged over the globe is 0.99 , and linearly regressing the APRPcomputed global-mean SW cloud feedback onto the kernel-computed global-mean SW cloud feedback yields a slope of 1.03 and an intercept of $0.02 \mathrm{~W} \mathrm{~m}^{-2}$ (not shown).

\section{b. Recently proposed metrics}

Observed and simulated values of the Sherwood metric (Table 1) are taken directly from Sherwood et al. (2014). The Sherwood metric is the sum of two mixing indices $S$ and $D$. The $S$ measures the climatological small-scale vertical mixing in the lower troposphere, defined as $S=\left[\left(\mathrm{RH}_{700 \mathrm{hPa}}-\mathrm{RH}_{850 \mathrm{hPa}}\right) / 100 \%-\right.$ $\left.\left(T_{700 \mathrm{hPa}}-T_{850 \mathrm{hPa}}\right) /(9 \mathrm{~K})\right] / 2$, where $T$ is air temperature. All quantities are taken from the corresponding climatologies averaged over tropical regions with climatological rising motion (primarily the western equatorial Pacific). A stronger lower-tropospheric mixing leads to a moister and colder free troposphere and hence a larger value of $S$. The $D$ measures the large-scale lowertropospheric mixing associated with the shallow overturning circulation. It is defined as

$$
D=\left\langle\left(\omega_{2}-\omega_{1}\right) H\left(\omega_{2}-\omega_{1}\right) H\left(-\omega_{1}\right)\right\rangle /\left\langle-\omega_{2} H\left(-\omega_{2}\right)\right\rangle,
$$

where $\omega_{1}$ is the average vertical velocity $\omega$ at 850 and $700 \mathrm{hPa} ; \omega_{2}$ is the average $\omega$ at 600,500 , and $400 \mathrm{hPa}$; $H$ is a step function; and angled brackets indicate the mean over tropical regions with climatological rising motion. A stronger shallow overturning circulation, corresponding to large $D$ values, generally leads to more large-scale lower-tropospheric mixing.

In Sherwood et al. (2014), the correlation between the Sherwood metric and ECS has been attributed to the physical connection between the metric and tropical low 
TABLE 3. The cross-model correlation between different metrics. Models with both metrics available (Table 1) are used to compute each correlation. The correlations whose $p$ values are less than 0.2 are deemed statistically significant and shown in boldface. Note that the signs of the Zhai and Tian metrics were reversed from their original values (as in Table 1) to compute the correlations.

\begin{tabular}{lccc}
\hline & Sherwood metric & Zhai metric & Su metric \\
\hline Zhai metric & 0.12 & - & - \\
Su metric & $\mathbf{0 . 5 1}$ & $\mathbf{0 . 5 0}$ & - \\
Tian metric & $\mathbf{0 . 7 1}$ & 0.09 & $\mathbf{0 . 4 9}$ \\
\hline
\end{tabular}

cloud feedback. Models with stronger climatological vertical moisture mixing in the lower troposphere (i.e., a larger Sherwood metric) are generally associated with a larger increase in the moisture mixing under climate change as the humidity contrast between free tropospheric and boundary layer air grows (Qu et al. 2015). Since the mixing dries the boundary layer and reduces boundary layer clouds, these models tend to have a larger decrease in boundary layer clouds under climate change, and therefore a more positive tropical low cloud feedback. This interpretation is also supported by a recent study performed with a multiparameter multiphysics model ensemble (Kamae et al. 2016).

The Tian metric (Table 1) is taken directly from Tian (2015). It is defined as the GCM bias (GCM minus observation) in regional-mean climatological precipitation in the southeastern Pacific $\left(30^{\circ} \mathrm{S}-0^{\circ}, 150^{\circ}-100^{\circ} \mathrm{W}\right)$. Given observational uncertainties in precipitation observations (Huffman et al. 2007; Adler et al. 2012), any values between -0.28 and $0.28 \mathrm{~mm} \mathrm{day}^{-1}$ are deemed realistic. This uncertainty range is computed as $20 \%$ of the observed regional-mean climatological precipitation in the southeastern Pacific (1.40 $\mathrm{mm} \mathrm{day}^{-1}$ ) (Tian 2015). The Tian metric is positive in almost all GCMs, consistent with the "double ITCZ" bias often found in GCMs. It turns out that the Tian metric is well correlated with the Sherwood metric $(r=0.71$; Table 3$)$. Apparently, models with greater climatological vertical mixing tend to have a smaller doubleITCZ bias. It is, however, unclear how to physically justify the relationship between the Tian and Sherwood metrics.

The Zhai metric (Table 1) is defined as the sensitivity of the regional-mean marine boundary layer cloud fraction to the regional-mean sea surface temperature (SST) in the context of the seasonal cycle (Zhai et al. 2015). It is computed by regressing the climatological monthly time series of the regional-mean marine boundary layer cloud fraction onto regional-mean SST. The regions of interest are the oceanic subsidence regions within $20^{\circ}-40^{\circ} \mathrm{N}$ and $20^{\circ}-40^{\circ} \mathrm{S}$. The metric is the average of the regression coefficients in the two hemispheres. Because some unconventional choices were made in Zhai et al. (2015), and to be consistent with other studies (Qu et al. 2014, 2015), we do not take the Zhai metric values directly from Zhai et al. (2015). Instead, we recompute the Zhai metric after employing several minor changes to the methodology. First, the height criterion for low clouds is changed from 700 to $680 \mathrm{hPa}$. Second, the number of model levels below the height criterion for low clouds is not fixed as in Zhai et al. (2015). Instead, at each location, we compute the pressure on all model levels, determine the number of model levels below the height criterion, and use those levels to compute the overall low cloud fraction. Note that the cloud-cover data in the CMIP5 archive is given on the native model levels, which vary with GCM. Third, the land-cover data provided by each GCM is used to identify ocean grid cells (i.e., land cover $<0.01 \%$ ). In Zhai et al. (2015), a common land-sea mask was first interpolated onto each model grid, and it was then used to define ocean grid cells in each GCM. These changes in methodology have little effect on the Zhai metric in almost all GCMs with the exception of three (CNRM-CM5, MIROC5, and NorESM1-M), where differences in the metric can be as large as a factor of 2. Nevertheless, the modified Zhai metric used throughout this paper is almost perfectly correlated with the original Zhai metric $(r=0.96)$.

In Zhai et al. (2015), the correlation between the Zhai metric and ECS has been attributed to the physical connection between the Zhai metric and tropical low cloud feedback. The latter was justified by the fact that the sensitivity of simulated low cloud cover (LCC) to SST is very similar in the contexts of the seasonal cycle and climate change. Namely, models with a more negative SST sensitivity in the context of the seasonal cycle tend to have a more negative SST sensitivity in the context of climate change. These models therefore exhibit a more positive tropical low cloud feedback. However, there is some ambiguity in interpreting these results, because SST is often anticorrelated with the strength of the tropical inversion [another important cloud-controlling factor, which is often measured by the estimated inversion strength (EIS)] (Wood and Bretherton 2006; Qu et al. 2014). It is unclear to what extent the Zhai metric was influenced by the seasonal relationship between LCC and EIS.

The Su metric (Table 1) is computed as the mean of the two metrics constructed in Su et al. (2014). The first metric $\alpha_{\mathrm{RH}}$ measures the similarity in the climatological zonal-mean RH between GCMs and observations. For each GCM, $\alpha_{\mathrm{RH}}$ is the regression slope of the simulated pattern of climatological zonal-mean $\mathrm{RH}$ between the surface and $100 \mathrm{hPa}$ and within $45^{\circ} \mathrm{S}-40^{\circ} \mathrm{N}$ onto the corresponding pattern in satellite (Aqua and Aura) observations. The second metric $\alpha_{\mathrm{CF}}$ measures the 
similarity in the climatological zonal-mean cloud fraction between GCMs and observations. It is obtained in a similar fashion as $\alpha_{\mathrm{RH}}$, except with CloudSat and CALIPSO observations. If the simulated and observed patterns match perfectly, both $\alpha_{\mathrm{RH}}$ and $\alpha_{\mathrm{CF}}$ would be 1 , yielding a value of 1 for their average (the Su metric) as well. However, given observational uncertainties in both $\mathrm{RH}$ and cloud fraction, any values of the Su metric between 0.75 and 1.25 are deemed realistic ( $\mathrm{Su}$ et al. 2014). In most GCMs, the Su metric is less than 0.75 (see Table 1 ), because of significant biases in the latitudinal and vertical distribution of $\mathrm{RH}$ and cloud fraction (Su et al. 2014). The Su metric is correlated with all other metrics (Table 3), but it is difficult to assign any physical significance to these correlations because of the small sample size of the metric.

The Zhai and Tian metrics are negatively correlated with ECS, while the Sherwood and Su metrics are positively correlated with ECS. For convenience, we reversed the signs of the Zhai and Tian metrics so all metrics are positively correlated with ECS.

\section{c. Artificial metrics}

As discussed in the introduction, any emergent constraint on ECS must satisfy at least two criteria: 1) it is significantly correlated with ECS, and 2) it is based on the observable characteristics of the current climate. Metrics meeting the first criterion can be generated statistically (see below). However, it is not straightforward to test whether these artificial metrics meet the second criterion. In this study, we treat these metrics as potential emergent constraints on ECS, with a collective behavior that is similar to the actual emergent constraints on ECS.

The artificial metrics are generated based on the following linear equation:

$$
M^{\prime}=a \mathrm{ECS}^{\prime}+b,
$$

where ECS' is the ECS anomaly for a particular GCM relative to the ensemble-mean normalized by the intermodel standard deviation of ECS, $a$ is a fixed number, and $b$ is a random number. Note that throughout the paper, primes denote standardized variables, as defined above. The $M^{\prime}$ is the standardized artificial metric we are generating. Here, we only know ECS', and our task is to choose appropriate values for $a$ and $b$ for each of the 26 GCMs so the $M^{\prime}$ resulting from Eq. (1) is correlated with $\mathrm{ECS}^{\prime}$ at 0.6 or above. The threshold correlation of 0.6 is chosen because it is the lowest of the correlations between the four existing metrics and ECS (Fig. 1).

Our method to construct $a$ and $b$ originates from simple regression analysis. According to such analysis (von Storch and Zwiers 1999), to construct a metric of 26 numbers that is correlated with $\mathrm{ECS}^{\prime}$ at a value of $a$, we need to find 26 values for $b$ such that the series formed by these 26 values has the mean of zero and standard deviation $\sigma$ of $\sqrt{1-a^{2}}$, and it is independent of ECS $\left[\operatorname{cov}\left(\mathrm{ECS}^{\prime}, b\right)=0\right]$. This can be verified in two steps. First, we can show that under those conditions, the $\sigma\left(M^{\prime}\right)$ resulting from Eq. (1) would be 1, as follows:

$$
\begin{aligned}
\sigma^{2}\left(M^{\prime}\right) & =a^{2} \sigma^{2}\left(\mathrm{ECS}^{\prime}\right)+\sigma^{2}(b)+2 a \operatorname{cov}\left(\mathrm{ECS}^{\prime}, b\right) \\
& =a^{2}+\left(1-a^{2}\right) \\
& =1
\end{aligned}
$$

Second, under the conditions $\sigma\left(M^{\prime}\right)=1, \sigma\left(\mathrm{ECS}^{\prime}\right)=1$, and $\operatorname{cov}\left(\mathrm{ECS}^{\prime}, b\right)=0$, we can express $r(M, \mathrm{ECS})$ as

$$
\begin{aligned}
r(M, \mathrm{ECS}) & =\operatorname{cov}\left(M^{\prime}, \mathrm{ECS}^{\prime}\right) \\
& =\operatorname{cov}\left(a \mathrm{ECS}^{\prime}+b, \mathrm{ECS}^{\prime}\right) \\
& =a \sigma^{2}\left(\mathrm{ECS}^{\prime}\right)+\operatorname{cov}\left(b, \mathrm{ECS}^{\prime}\right) \\
& =a .
\end{aligned}
$$

The derivations above show that it is possible to construct an artificial metric correlated with ECS at a predetermined value $a$, if we can find the right $b$ values. Following this approach, we set $a$ to each of the four values $(0.6,0.7,0.8$, or 0.9$)$ in Eq. (1) successively. In each case, we choose a $b$ value for each GCM from a random variable with a normal distribution, which has the mean of zero and standard deviation of $\sqrt{1-a^{2}}$. It turns out that the series formed by the 26 values of $b$ constructed as described above is generally not independent of $\mathrm{ECS}^{\prime}$, because of random sampling. So, the correlation between ECS ${ }^{\prime}$ and $M^{\prime}$ constructed with Eq. (1) generally deviates from $a$. To sample enough values of $r(M, \mathrm{ECS})$ around $a$, for each value of $a$ we repeat the procedures described above 1000 times to get 1000 metrics. We have in total 4000 metrics. Out of these 4000 metrics, 577 yield values of $r(M, \mathrm{ECS})$ less than 0.6 , and are hence discarded. Values of $r(M, \mathrm{ECS})$ for the remaining 3423 metrics range from 0.60 to 0.97 , with the mean of 0.80 and standard deviation of 0.10 . Note that the sample size 3423 is large enough to provide stable statistics, and any increase in this number has little effect on the results.

\section{d. An analytical framework for the correlation between a metric and ECS}

To diagnose what drives the correlation between each metric $M$ and ECS $[r(M$, ECS $)]$, we start with the definition of $r(M, \mathrm{ECS})$ (von Storch and Zwiers 1999):

$$
r(M, \mathrm{ECS})=\operatorname{cov}\left(M^{\prime}, \mathrm{ECS}^{\prime}\right),
$$


TABLE 4. The cross-model correlation coefficient between SW cloud feedback and other feedbacks or forcing in 26 CMIP5 GCMs (Table 1). The correlations whose $p$ values are less than 0.2 are deemed statistically significant and shown in boldface.

\begin{tabular}{cccccccc}
\hline \hline & $F$ & $\lambda_{\mathrm{Pl}}$ & $\lambda_{\mathrm{WV}}$ & $\lambda_{\mathrm{LR}}$ & $\lambda_{\text {LWcld }}$ & $\lambda_{\mathrm{Alb}}$ & $\lambda_{\mathrm{Re}}$ \\
\hline$\lambda_{\text {SWcld }}$ & $\mathbf{- 0 . 5 6}$ & -0.12 & $\mathbf{- 0 . 3 6}$ & 0.06 & $\mathbf{- 0 . 6 2}$ & 0.13 & -0.19 \\
\hline
\end{tabular}

where $M^{\prime}$ and $\mathrm{ECS}^{\prime}$ are the standardized anomalies as defined in section 2c.

To connect $M^{\prime}$ with the various factors contributing to ECS spread, we project $M^{\prime}$ onto different feedbacks and forcing by performing multiple regression analysis, as follows:

$$
M^{\prime}=\sum \beta_{i} X_{i}^{\prime}+\varepsilon,
$$

where $X_{i}^{\prime}$, defined as a standardized anomaly similar to $M^{\prime}$, is taken from $X^{\prime}=\left\{F^{\prime}, \lambda_{\mathrm{Pl}}^{\prime}, \lambda_{\mathrm{WV}}^{\prime}, \lambda_{\mathrm{LR}}^{\prime}, \lambda_{\mathrm{SWcld}}^{\prime}\right.$, $\left.\lambda_{\mathrm{LWcld}}^{\prime}, \lambda_{\mathrm{Alb}}^{\prime}, \lambda_{\mathrm{Re}}^{\prime}\right\}$. The regression coefficients $\beta_{i}$ in Eq. (5) quantify the intermodel variance of $M$ associated with $X_{i}^{\prime}$. To identify the variables in $X^{\prime}$ that have a significant relationship with $M^{\prime}$, a backward selection process is used in conjunction with the multiple regression analysis (Neter et al. 1996). First, we use a regression model containing all eight variables in $X^{\prime}$ and identify the variable with the largest $p$ value. If the two-tailed $p$ value associated with this variable is greater than a threshold (typically, 0.2), it is assumed that this variable has no significant relationship with $M^{\prime}$. (The condition that the $p$ value is less than 0.2 is equivalent to the condition that the 10th-90th percentile of the associated regression coefficient estimate does not span zero.) Then this variable is eliminated from the original regression model, and the analysis is repeated with a regression model containing the seven remaining variables. This procedure is repeated until all $p$ values in the regression model are less than the threshold value. The final regression model is then used in the subsequent analysis. The backward selection preferentially removes factors whose weak statistical link to the metric arises from covariability with factors exhibiting a stronger relationship with the metric. Covariability arises because the variables in $X^{\prime}$ are generally not independent of one another (Caldwell et al. 2016; see also Table 4). Here, we assume that those factors with the stronger statistical relationship with the metric are more likely to have a physical relationship with the metric. The last term in Eq. (5) $\varepsilon$ represents the residual of $M^{\prime}$ in the final regression model.

Plugging Eq. (5) into Eq. (4), we obtain an analytical expression for $r(M, \mathrm{ECS})$ :

$$
\begin{aligned}
r(M, \mathrm{ECS}) & =\operatorname{cov}\left(M^{\prime}, \mathrm{ECS}^{\prime}\right) \\
& =\operatorname{cov}\left(\sum \beta_{i} X_{i}^{\prime}+\varepsilon, \mathrm{ECS}^{\prime}\right) \\
& =\sum \beta_{i} \operatorname{cov}\left(X_{i}^{\prime}, \mathrm{ECS}^{\prime}\right)+\operatorname{cov}\left(\varepsilon, \mathrm{ECS}^{\prime}\right) \\
& =\sum \beta_{i} r\left(X_{i}, \mathrm{ECS}\right)+\operatorname{cov}\left(\varepsilon, \mathrm{ECS}^{\prime}\right) .
\end{aligned}
$$

The first term on the right-hand side of Eq. (6) represents the sum of the contributions to $r(M, \mathrm{ECS})$ by the various factors $X_{i}$ identified by the backward selection process. Each contribution is the product of $\beta_{i}$ and the correlation between $X_{i}$ and ECS. The second represents the residual term of $r(M, \mathrm{ECS})$. As shown later, the residual term is generally small, so Eq. (6) is adequate to diagnose the origins of correlations between ECS and the various metrics described above.

\section{Results}

Figure 2a shows values of $\beta_{i}$ for the four recently proposed metrics. SW cloud feedback is very significantly related to all metrics, with $p$ values (Table 5) two orders of magnitude less than the threshold value (0.2) used in the backward selection process. The relationships between SW cloud feedback and the Sherwood and Zhai metrics are reassuring, as they are what motivated the two metrics in the first place (Sherwood et al. 2014; Zhai et al. 2015). However, the physics behind the connection between SW cloud feedback and the two other metrics ( $\mathrm{Su}$ and Tian) are less clear. Besides SW cloud feedback, we identify three other factors significantly related to the metrics, including the forcing term $F, \lambda_{\mathrm{LWcld}}$, and $\lambda_{\mathrm{Re}}$ (see Fig. 2a). However, the $p$ values associated with those factors are generally larger than those of SW cloud feedback (Table 5).

The correlations (Table 6) and regression coefficients (Fig. 2a) associated with all factors are positive. The regression coefficients associated with SW cloud feedback are generally greater than those associated with other factors, and all four metrics are also best correlated with SW cloud feedback (Table 6). The intermodel variance in each metric that is accounted for by all factors combined is typically $60 \%-70 \%$ of the total variance in that metric (not shown). In Fig. $2 \mathrm{~b}$ we show the other quantities determining $r(M, \mathrm{ECS})$ in Eq. (6), namely, $r\left(X_{i}\right.$, ECS $)$, or the correlation between each ECS-controlling factor $X_{i}$ and ECS. In all cases, SW cloud feedback exhibits the strongest correlation with ECS, consistent with the fact that this feedback is the leading source of ECS spread in the current generation of GCMs (Table 2) (Andrews et al. 2012; Caldwell et al. 2016). 
(a) $\beta$ : Relationships of the four metrics with eight factors

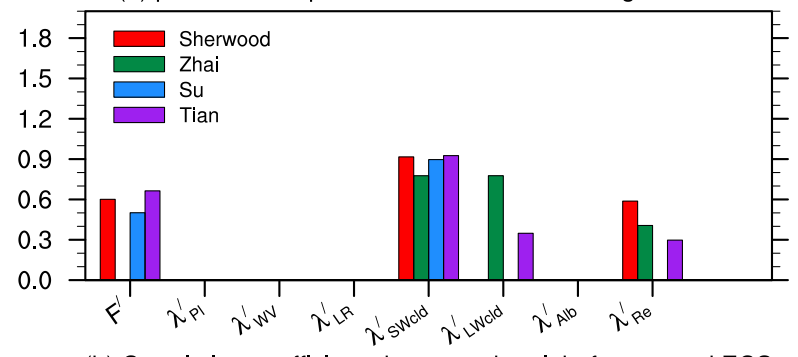

(b) Correlation coefficients between the eight factors and ECS

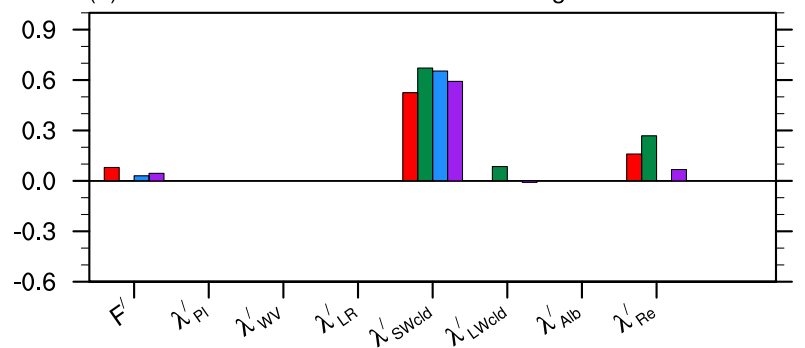

(c) Contributions of the eight factors to $r(M, E C S)$

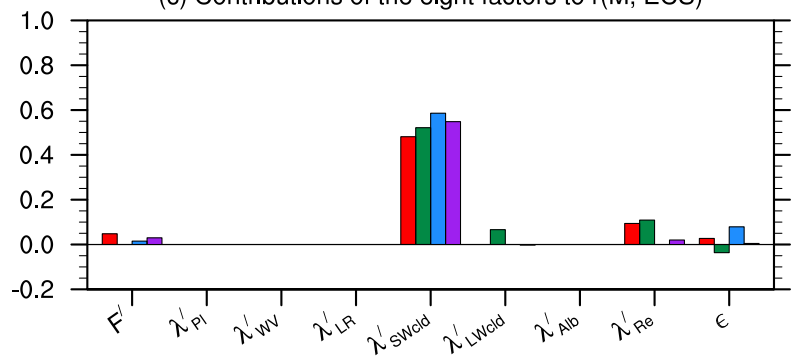

FIG. 2. Contributions of the eight factors influencing ECS to the correlations $r$ between the four recently proposed metrics and ECS. (a) The relationships of the four metrics with the eight factors, quantified by the regression coefficients $\beta_{i}$ in Eq. (6). See methods for the definition of factors. (b) Correlation coefficients between the eight factors and ECS. The correlations associated with a particular factor differ slightly for the different metrics because the GCMs included in the calculations vary with the metrics (Table 1). (c) Contributions of the eight factors to the correlations between the four metrics and ECS, quantified based on Eq. (6). The portion of $r(M, \mathrm{ECS})$ unaccounted for by the eight factors, namely, the $\varepsilon$ term of Eq. (6), is also shown in (c). Note that we only show the results for the combinations of factor and metric that are significantly related according to the backward selection process.

The final values of the various components of $r(M, \mathrm{ECS})$ [i.e., terms on the right-hand side of Eq. (6)] are shown in Fig. 2c. For all metrics, the SW cloud feedback term emerges as by far the dominant contributor to $r(M, \mathrm{ECS})$. This dominance occurs because $r\left(\lambda_{\text {SWcld }}\right.$, ECS $)$ is much larger than the other $r$ terms (Fig. 2b), and the regression coefficients associated with SW cloud feedback are somewhat larger than the other regression coefficients (Fig. 2a). Note that the contribution of $\varepsilon$ [the last term of $r(M, \mathrm{ECS})$ in Eq. (6)] is generally small, serving as a validation of our linear methodology.
TABLE 5. The $p$ values associated with the various factors that have a significant relationship with a particular metric according to the backward selection. Note that the number of the GCMs used in these calculations differs ( 26 for the Sherwood metric, 15 for the Zhai metric, 13 for the Su metric, and 25 for the Tian metric; see Table 1).

\begin{tabular}{lcrcc}
\hline \hline & $F$ & $\lambda_{\text {SWcld }}$ & $\lambda_{\text {LWcld }}$ & \multicolumn{1}{c}{$\lambda_{\text {Re }}$} \\
\hline Sherwood & $<0.001$ & $<0.001$ & - & $<0.001$ \\
Zhai & - & 0.004 & 0.004 & 0.049 \\
Su & 0.058 & 0.003 & - & - \\
Tian & 0.003 & $<0.001$ & 0.101 & 0.079 \\
\hline
\end{tabular}

Given the strong connection between the metrics and SW cloud feedback, we examine how this connection manifests itself geographically by correlating each metric with a measure of local SW cloud feedback at each grid point across GCMs (Figs. 3a-d). For all metrics, regions with the largest correlations are primarily the tropical oceanic subsidence regions dominated by low clouds. This lends credence to hypotheses put forward in Sherwood et al. (2014) and Zhai et al. (2015), namely, that correlations between these two metrics and ECS are attributable to the connection between the metrics and tropical low cloud feedback. Similar geographic distributions are also seen in the correlation of each metric with the two components of SW cloud feedback obtained via APRP, namely, the cloud amount (Fig. 4) and scattering plus absorption feedbacks (Fig. 5).

Since SW cloud feedback is the dominant contributor to $r(M, \mathrm{ECS})$, it is reasonable to hypothesize that the generally large ECS values in GCMs deemed realistic (i.e., those lying in the gray area of each diagram in Fig. 1) stem from a SW cloud feedback that is more positive than the ensemble mean. This hypothesis can be evaluated by examining the standardized anomalies in SW cloud feedback $\lambda_{\text {SWcld }}^{\prime}$ in the GCMs deemed realistic according to each metric. Figure 6 shows values of $\lambda_{\text {SWcld }}^{\prime}$ in those GCMs (symbols) and the full range of $\lambda_{\text {SWcld }}^{\prime}$ (whiskers) among all models. Note that the number of

TABLE 6 . The cross-model correlation coefficients between the metrics and different factors and the associated $p$ values (in parentheses). The correlations whose $p$ values are less than 0.2 are deemed statistically significant and shown in boldface. Note that we only show the results for the combinations of factor and metric that are significantly related according to the backward selection process.

\begin{tabular}{lcccc}
\hline \hline & $F$ & $\lambda_{\text {SWcld }}$ & $\lambda_{\text {LWcld }}$ & $\lambda_{\text {Re }}$ \\
\hline Sherwood & $0.03(0.884)$ & $\mathbf{0 . 4 7}(0.015)$ & - & $\mathbf{0 . 3 5}(0.080)$ \\
Zhai & - & $\mathbf{0 . 3 9}(0.151)$ & $0.27(0.330)$ & $0.31(0.261)$ \\
Su & $0.04(0.897)$ & $\mathbf{0 . 6 4}(0.018)$ & - & - \\
Tian & $\mathbf{0 . 2 9}(0.160)$ & $\mathbf{0 . 3 0}(0.145)$ & $0.15(0.474)$ & $0.08(0.704)$ \\
\hline
\end{tabular}




\section{Correlation coefficients between different metrics and SW cloud feedback}

(a) Sherwood metric

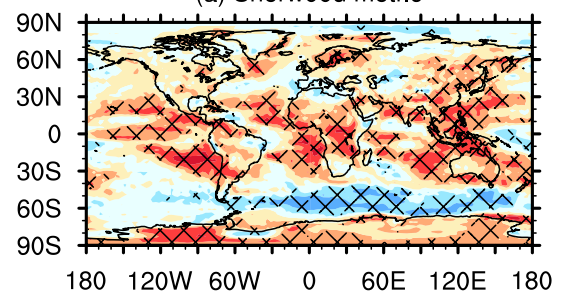

(b) Zhai metric

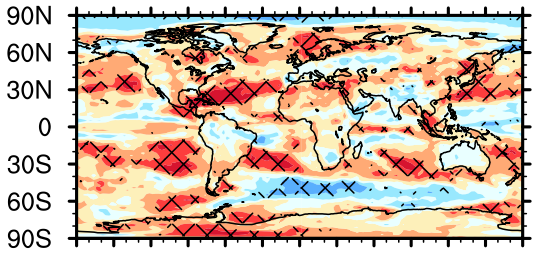

(c) Su metric

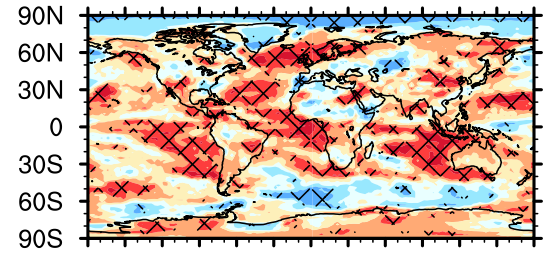

180 120W 60W $0 \quad 60 \mathrm{E} \quad 120 \mathrm{E} \quad 180$

(d) Tian metric

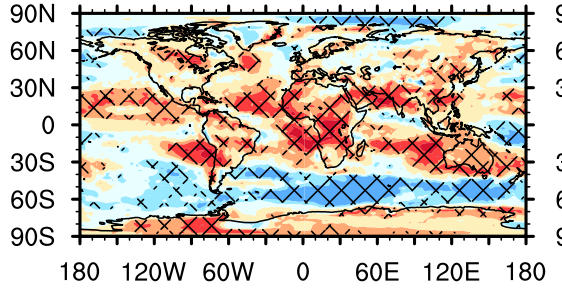

(e) Artificial metrics
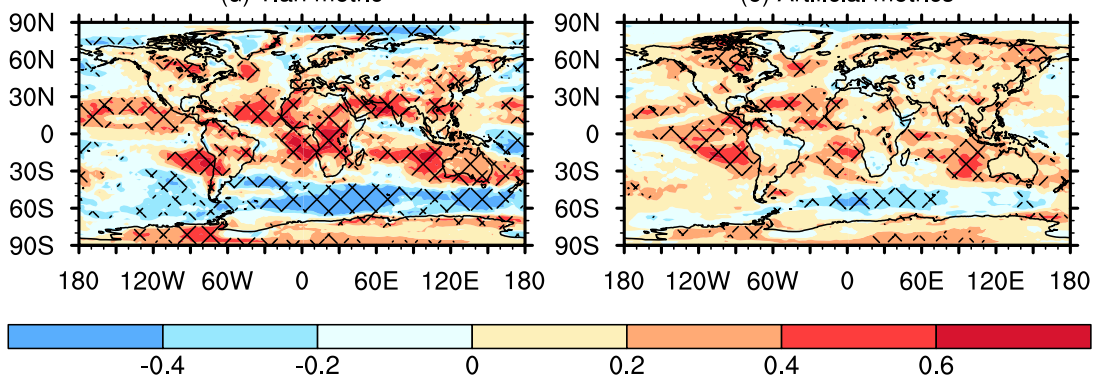

FIG. 3. Geographic distribution of the cross-model correlation coefficient between different metrics and SW cloud feedback: (a) Sherwood metric, (b) Zhai metric, (c) Su metric, (d) Tian metric, and (e) artificial metrics. To obtain (a)-(d), we correlate each metric with a measure of local SW cloud feedback at each grid point across GCMs. The local SW cloud feedback is obtained by applying the Gregory method to the SW flux at each grid point. To obtain (e), we first correlate each artificial metric with the local SW cloud feedback at each grid point across GCMs and then average the correlations over all artificial metrics. In each diagram, the correlations whose $p$ values are less than 0.2 are hatched. Note that the number of the GCMs used in these calculations differs (see Table 1).

realistic GCMs varies with metrics (5 for the Sherwood, Zhai, and Su metrics and 1 for the Tian metric; see Fig. 1). For comparison, the standardized anomalies in other feedback and forcing terms are also shown in Fig. 6. In the cases of the Sherwood (the red crosses in Fig. 6) and $\mathrm{Su}$ (the blue crosses) metrics, the anomalies in SW cloud feedback are predominantly positive in the realistic GCMs. In the case of the Zhai metric (the green crosses), $60 \%$ of the realistic GCMs exhibit positive anomalies in SW cloud feedback. The only GCM identified by the Tian

\section{Correlation coefficients between different metrics and SW cloud amount feedback}
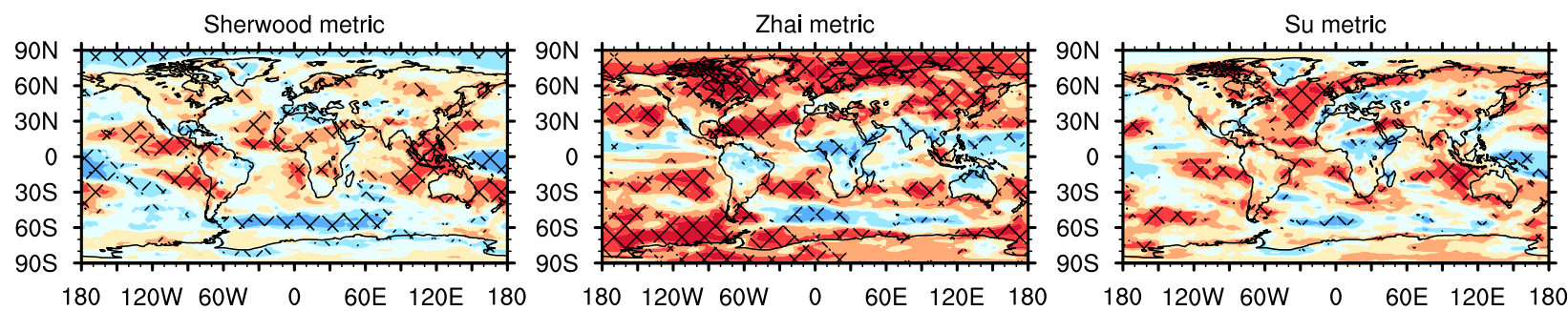

180 120W 60W 0 60E $120 \mathrm{E} \quad 180$

180 120W 60W $0 \quad 60 \mathrm{E} \quad 120 \mathrm{E} \quad 180$

$180120 \mathrm{~W} 60 \mathrm{~W}$

$0 \quad 60 \mathrm{E} \quad 120 \mathrm{E} \quad 180$ Tian metric
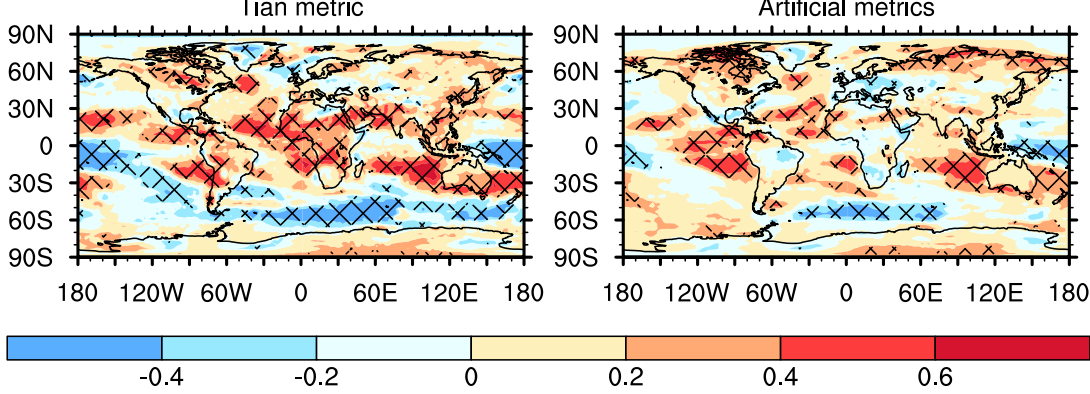

FIG. 4. As in Fig. 3, but for the APRP-derived SW cloud amount feedback. 


\section{Correlation coefficients between different metrics and SW cloud scattering+absorption feedback}
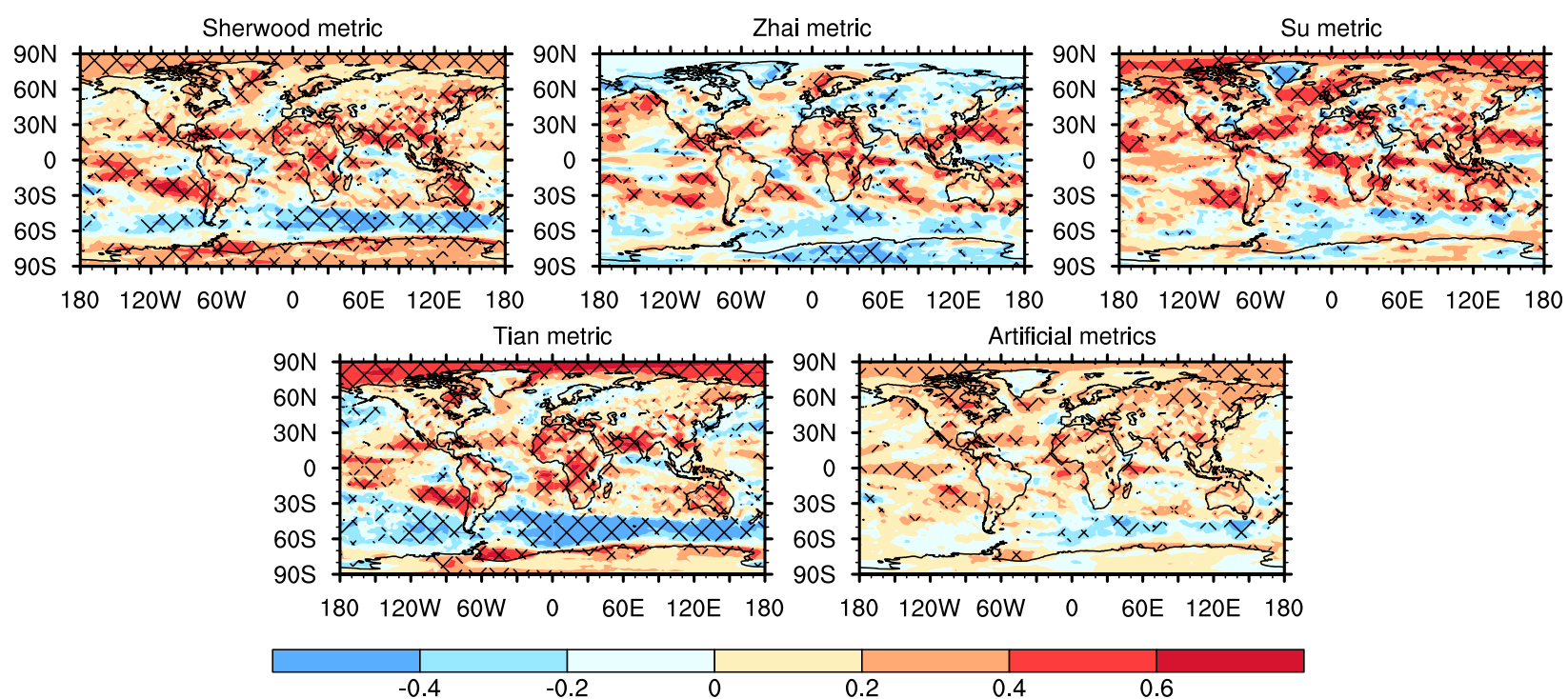

FIG. 5. As in Fig. 3, but for the APRP-derived SW cloud scattering-plus-absorption feedback.

metric as "realistic" (the purple crosses) has a positive anomaly in SW cloud feedback as well. These results lead credence to our hypothesis. However, several realistic GCMs have negative anomalies in SW cloud feedback, and the high ECS values in those GCMs stem from positive anomalies in other feedback or forcing terms (Fig. 6). Since these terms contribute little to the metricECS correlation (Fig. 2c), the high ECS predictions by the metrics are difficult to interpret. Note that because the number of realistic GCMs is relatively small, some of the results may be influenced by random sampling.

Figure 7a shows the statistics of $\beta_{i}$ associated with the artificial metrics. The artificial metrics exhibit many similar features as the recently proposed metrics (cf. Figs. $2 \mathrm{a}$ and $7 \mathrm{a}$ ). First, all artificial metrics are significantly related to SW cloud feedback. This connection typically originates in the subsidence regions dominated by low clouds (cf. Figs. 3a-d and 3e). The metrics are typically correlated with both SW cloud amount feedback (cf. Figs. 4a-d and 4e) and SW cloud scattering plus absorption feedback (cf. Figs. $5 \mathrm{a}-\mathrm{d}$ and $5 \mathrm{e}$ ) in those regions. Second, the typical $\beta$ value associated with SW cloud feedback is larger than those associated with other factors (comparing the values in Fig. 7a). Finally, other than SW cloud feedback, $F, \lambda_{\text {LWcld }}$, and $\lambda_{\operatorname{Re}}$ are also frequently identified as significant by the backward selection process (Fig. 7a). The correlation between ECS and each feedback or forcing, $r\left(X_{i}\right.$, ECS) in Eq. (6), is shown in Fig. 7b, and the various terms of Eq. (6) for the artificial metrics are shown in Fig. 7c. As in the case of recently proposed metrics, the SW cloud feedback term emerges as by far the dominant contributor to the correlations between almost all artificial metrics and ECS (Fig. 7c).

In summary, we demonstrate that 1) both the recently proposed and all the artificial metrics are significantly related to SW cloud feedback and 2) such a relationship is the main driver of the correlations between those metrics and ECS. The importance of SW cloud feedback for the metric-ECS relationship is rooted in the fact that SW cloud feedback is the leading source of ECS spread (Table 2). However, there may be other reasons for the dominance of the SW cloud feedback term over other terms in Eq. (6). For example, the anticorrelations between SW cloud feedback and several other factors (Table 4) may diminish terms associated with those factors (Figs. $2 \mathrm{c}$ and $7 \mathrm{c}$ ) by suppressing their correlations with ECS (Figs. 2b and 7b). Take LW cloud feedback as an example. This feedback exhibits a substantial spread (Table 2), but little correlation with ECS (Figs. 2b and $7 b)$, probably because its contribution to ECS spread is largely canceled out by the contribution of SW cloud feedback because of the anticorrelation between the two. So this feedback contributes little to the correlations between the metrics and ECS (Figs. 2c and 7c).

\section{Concluding remarks}

Our results suggest that the four proposed constraints on ECS may be reframed as potential constraints on SW low cloud feedback. Of course, here we did not examine physical links between the metrics and SW low cloud 


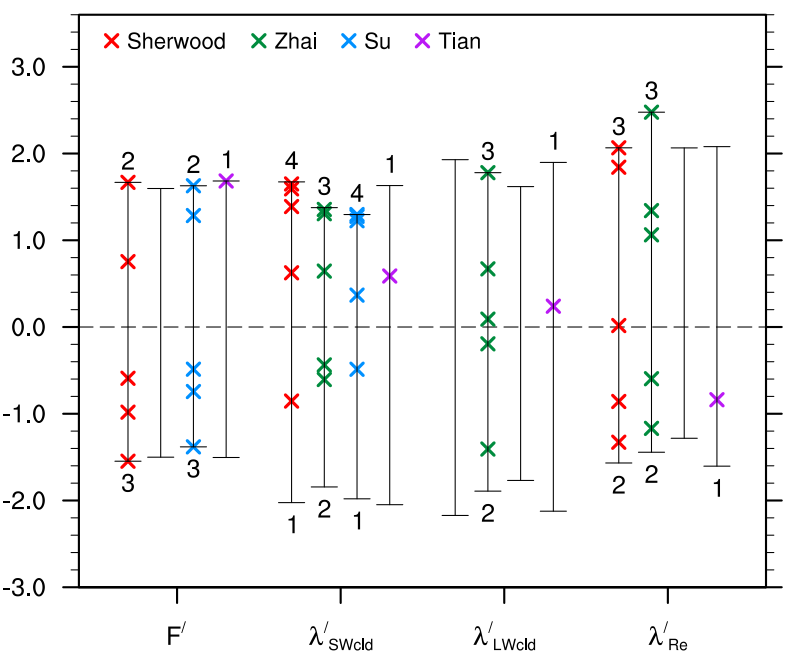

FIG. 6. The standardized anomalies in various feedbacks and forcing for GCMs deemed realistic by the Sherwood, Zhai, Su, or Tian metric (see Fig. 1). The standardized anomalies are the intermodel anomalies normalized by the intermodel standard deviation. GCMs are color-coded in red, green, blue, or purple depending on whether the anomalies are computed using the available Sherwood, Zhai, Su, or Tian GCMs, respectively. We only show the results for the factors significantly related to at least one metric. The associated range (including the minimum and maximum) of the anomalies in all Sherwood, Zhai, Su, or Tian GCMs are also shown as the whisker. For each factor, the number of realistic GCMs with a positive anomaly is shown above the associated range, while the number of realistic GCMs with a negative anomaly is shown below the associated range.

feedback. To become true emergent constraints, further investigation of the connection between the metrics and SW low cloud feedback would be necessary, especially for those metrics lacking an obvious physical connection with low clouds (e.g., the Su and Tian metrics). Furthermore, since the proposed constraints are unlikely to constrain factors other than SW low cloud feedback (Fig. 6), and since these other factors could be systematically biased in GCMs, the proposed constraints should be viewed with caution. Indeed, several recent studies demonstrate the importance of other factors possibly unrelated to tropical low cloud feedback in driving model spread in ECS. These include deep convection precipitation microphysics, mixed-phase cloud physics, and cumulus congestus and anvil cloud feedbacks (Zhao et al. 2016; Tan et al. 2016; Kamae et al. 2016; Mauritsen and Stevens 2015; Bony et al. 2016). To put a useful constraint on ECS, all these factors should be constrained individually (Klein and Hall 2015).

According to our findings, any metric correlated with ECS in the CMIP5 model ensemble is most likely to be statistically linked to SW cloud feedback via tropical low cloud feedback. This suggests that other emergent constraints not included in this study, including any that may (a) $\beta$ : Relationships of the artificial metrics with eight factors

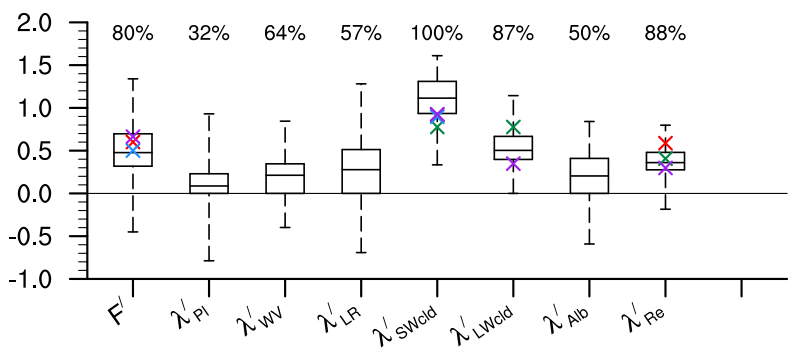

(b) Correlation coefficients between the eight factors and ECS

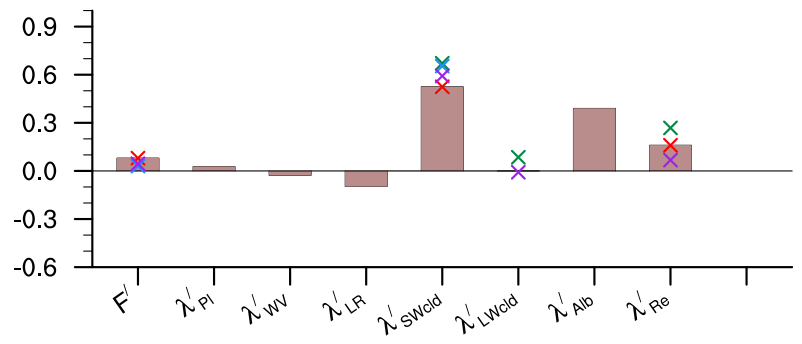

(c) Contributions of the eight factors to $r\left(M_{a}, E C S\right)$

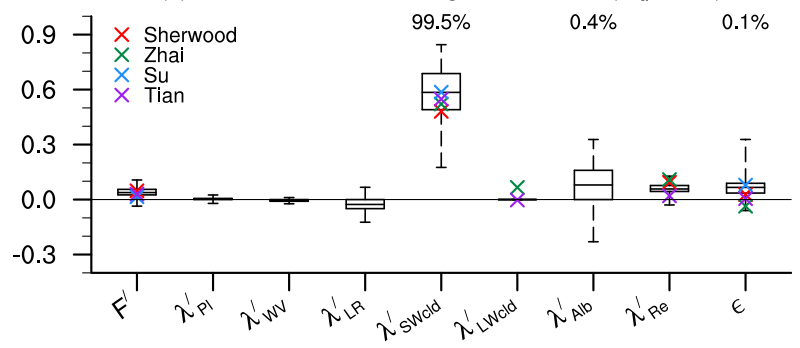

FIG. 7. As in Fig. 2, but for the artificial metrics $M_{a}$. (a) The relationships of $M_{a}$ with the eight factors influencing ECS, quantified by the regression coefficients $\beta_{i}$ in Eq. (6). The distribution of $\beta_{i}$ for all artificial metrics (including the minimum, 25th percentile, mean, 75th percentile, and maximum) is represented by the box-andwhisker plots. We set $\beta_{i}$ to zero for all factors exhibiting no significant relationship with each artificial metric. The portion of the artificial metrics exhibiting a significant relationship with each factor is also shown. (b) Correlation coefficients (represented by the bars) between the eight factors and ECS using all 26 GCMs. (c) Contributions of the eight factors to $r\left(M_{a}\right.$, ECS $)$, quantified based on Eq. (6). The $\lambda_{\text {SWcld }}^{\prime}$ term makes the greatest contribution for $99.5 \%$ of the artificial metrics (the number shown above the whisker), while the $\lambda_{\mathrm{Alb}}^{\prime}$ and $\varepsilon$ terms make the greatest contributions for $0.4 \%$ and $0.1 \%$ of the artificial metrics, respectively. For comparison, the respective values for the four recently proposed metrics (as in Fig. 2) are represented by the crosses on all panels.

be proposed in the future, may also be linked to tropical low cloud feedback just like the four recent emergent constraints. Since those other emergent constraints not included in this study may be statistically indistinguishable from the four recent emergent constraints examined in this study, and since all but one of the 11 published emergent constraints for ECS predict a high ECS value, odds favor that future emergent constraints would also predict a high ECS value. [High ECS is 
predicted by 10 constraints in nine studies (Volodin 2008; Trenberth and Fasullo 2010; Fasullo and Trenberth 2012; Sherwood et al. 2014; Su et al. 2014; Tian 2015; Zhai et al. 2015; Brient and Schneider 2016; Siler et al. 2018), whereas Lipat et al. (2017) offers the only constraint that predicts low ECS.] A caveat is that ECS spread in future model ensembles could be driven by factors other than tropical low cloud feedback. In such ensembles, the emergent constraints of ECS may be related to other factors influencing ECS. However, we deem this possibility unlikely in a multimodel ensemble like CMIP5, given the continuing uncertainty surrounding tropical low cloud feedback.

Acknowledgments. X. Qu, A. Hall, A. M. DeAngelis, M. D. Zelinka, and S. A. Klein are supported by the DOE's Regional and Global Climate Modeling Program under the project "Identifying Robust Cloud Feedbacks in Observations and Models." X. Qu and A. Hall are also supported by NSF Grant 1543268 , titled "Reducing Uncertainty Surrounding Climate Change Using Emergent Constraints." The work of M. D. Zelinka and S. A. Klein was performed under the auspices of the U.S. Department of Energy by Lawrence Livermore National Laboratory under Contract DE-AC52-07NA27344. The work of H. Su, B. Tian, and C. Zhai was performed at Jet Propulsion Laboratory (JPL), California Institute of Technology, under contract with NASA. H. Su and C. Zhai are supported by the NASA NEWS program. We acknowledge the modeling groups, the Program for Climate Model Diagnosis and Intercomparison (PCMDI), and the WCRP's Working Group on Coupled Modelling (WGCM) for their roles in making available the WCRP CMIP3 and CMIP5 multimodel datasets. Support of these datasets is provided by the Office of Science, U.S. Department of Energy. We thank all reviewers for their positive comments and constructive feedback on our manuscript and Peter Caldwell and Chen Zhou for discussion on the topic. (The Sherwood metric was downloaded from https://www.nature.com/nature/journal/v505/ n7481/full/nature12829.html.)

\section{REFERENCES}

Adler, R. F., G. Gu, and G. J. Huffman, 2012: Estimating climatological bias errors for the Global Precipitation Climatology Project (GPCP). J. Appl. Meteor. Climatol., 51, 84-99, https:// doi.org/10.1175/JAMC-D-11-052.1.

Andrews, T., J. M. Gregory, M. J. Webb, and K. E. Taylor, 2012: Forcing, feedbacks and climate sensitivity in CMIP5 coupled atmosphere-ocean climate models. Geophys. Res. Lett., 39, L09712, https://doi.org/10.1029/2012GL051607.

,-- , and,- 2015 : The dependence of radiative forcing and feedback on evolving patterns of surface temperature change in climate models. J. Climate, 28, 1630-1648, https:// doi.org/10.1175/JCLI-D-14-00545.1.
Bony, S., and J.-L. Dufresne, 2005: Marine boundary layer clouds at the heart of tropical cloud feedback uncertainties in climate models. Geophys. Res. Lett., 32, L20806, https://doi.org/ 10.1029/2005GL023851.

- B. Stevens, D. Coppin, T. Becker, K. A. Reed, A. Voigt, and B. Medeiros, 2016: Thermodynamic control of anvil cloud amount. Proc. Natl. Acad. Sci. USA, 113, 8927-8932, https:// doi.org/10.1073/pnas.1601472113.

Bretherton, C. S., 2015: Insights into low-latitude cloud feedbacks from high-resolution models. Philos. Trans. Roy. Soc., 373A, 20140415, https://doi.org/10.1098/rsta.2014.0415.

Brient, F., and T. Schneider, 2016: Constraints on climate sensitivity from space-based measurements of low-cloud reflection. J. Climate, 29, 5821-5835, https://doi.org/10.1175/JCLI-D-15-0897.1.

Caldwell, P. M., M. D. Zelinka, K. E. Taylor, and K. Marvel, 2016: Quantifying the sources of intermodel spread in equilibrium climate sensitivity. J. Climate, 29, 513-524, https://doi.org/ 10.1175/JCLI-D-15-0352.1.

Ceppi, P., D. T. McCoy, and D. L. Hartmann, 2016: Observational evidence for a negative shortwave cloud feedback in middle to high latitudes. Geophys. Res. Lett., 43, 1331-1339, https:// doi.org/10.1002/2015GL067499.

Clement, A. C., R. Burgman, and J. R. Norris, 2009: Observational and model evidence for positive low-level cloud feedback. Science, 325, 460-464, https://doi.org/10.1126/science.1171255.

Collins, M., and Coauthors, 2013: Long-term climate change: Projections, commitments and irreversibility. Climate Change 2013: The Physical Science Basis, T. F. Stocker et al., Eds., Cambridge University Press, 1029-1136.

Cubasch, U., and Coauthors, 2001: Projections of future climate change. Climate Change 2001: The Scientific Basis, J. T. Houghton et al., Eds., Cambridge University Press, 525-582.

DeAngelis, A. M., X. Qu, M. D. Zelinka, and A. Hall, 2015: An observational radiative constraint on hydrologic cycle intensification. Nature, 528, 249-253, https://doi.org/10.1038/nature15770.

Fasullo, J. T., and K. E. Trenberth, 2012: A less cloudy future: The role of subtropical subsidence in climate sensitivity. Science, 338, 792-794, https://doi.org/10.1126/science.1227465.

Gordon, N. D., and S. A. Klein, 2014: Low-cloud optical depth feedback in climate models. J. Geophys. Res. Atmos., 119, 6052-6065, https://doi.org/10.1002/2013JD021052.

Gregory, J. M., and Coauthors, 2004: A new method for diagnosing radiative forcing and climate sensitivity. Geophys. Res. Lett., 31, L03205, https://doi.org/10.1029/2003GL018747.

Held, I. M., and K. M. Shell, 2012: Using relative humidity as a state variable in climate feedback analysis. J. Climate, 25, 25782582, https://doi.org/10.1175/JCLI-D-11-00721.1.

Huffman, G. J., and Coauthors, 2007: The TRMM Multisatellite Precipitation Analysis (TMPA): Quasi-global, multiyear, combined-sensor precipitation estimates at fine scales. J. Hydrometeor., 8, 38-55, https://doi.org/10.1175/JHM560.1.

Kamae, Y., H. Shiogama, M. Watanabe, T. Ogura, T. Yokohata, and M. Kimoto, 2016: Lower-tropospheric mixing as a constraint on cloud feedback in a multiparameter multiphysics ensemble. J. Climate, 29, 6259-6275, https://doi.org/10.1175/ JCLI-D-16-0042.1.

Kattenberg, A., and Coauthors, 1996: Climate models-Projections of future climate. Climate Change 1995: The Science of Climate Change, J. T. Houghton et al., Eds., Cambridge University Press, 285-357.

Klein, S. A., and A. Hall, 2015: Emergent constraints for cloud feedbacks. Curr. Climate Change Rep., 1, 276-287, https:// doi.org/10.1007/s40641-015-0027-1. 
Lipat, B. R., G. Tselioudis, K. M. Grise, and L. M. Polvani, 2017: CMIP5 models' shortwave cloud radiative response and climate sensitivity linked to the climatological Hadley cell extent. Geophys. Res. Lett., 44, 5739-5748, https://doi.org/ 10.1002/2017GL073151.

Lu, J., G. A. Vecchi, and T. Reichler, 2007: Expansion of the Hadley cell under global warming. Geophys. Res. Lett., 34, L06805, https://doi.org/10.1029/2006GL028443.

Mauritsen, T., and B. Stevens, 2015: Missing iris effect as a possible cause of muted hydrological change and high climate sensitivity in models. Nat. Geosci., 8, 346-351, https://doi.org/ 10.1038/ngeo2414.

Meehl, G. A., and Coauthors, 2007: Global climate projections. Climate Change 2007: The Physical Science Basis, S. Solomon et al., Eds., Cambridge University Press, 747-846.

Mitchell, J. F. B., S. Manabe, V. Meleshko, and T. Tokioka, 1990: Equilibrium climate change-And its implications for the future. Climate Change: The IPCC Scientific Assessment, J. T. Houghton, G. J. Jenkins, and J. J. Ephraums, Eds., Cambridge University Press, 131-172, https://www.ipcc.ch/ipccreports/ far/wg_I/ipcc_far_wg_I_chapter_05.pdf.

Neter, J., M. H. Kutner, C. J. Nachtsheim, and W. Wasserman, 1996: Building the regression model I: Selection of predictor variables. Applied Linear Statistical Models, 4th ed. WCB/ McGraw-Hill, 327-360.

Qu, X., A. Hall, S. A. Klein, and P. M. Caldwell, 2014: On the spread of changes in marine low cloud cover in climate model simulations of the 21st century. Climate Dyn., 42, 2603-2626, https://doi.org/10.1007/s00382-013-1945-z.

$\longrightarrow,-, \ldots$, and A. M. DeAngelis, 2015: Positive tropical marine low-cloud cover feedback inferred from cloudcontrolling factors. Geophys. Res. Lett., 42, 7767-7775, https://doi.org/10.1002/2015GL065627.

Shell, K. M., J. T. Kiehl, and C. A. Shields, 2008: Using the radiative kernel technique to calculate climate feedbacks in NCAR's Community Atmospheric Model. J. Climate, 21, 2269-2282, https://doi.org/10.1175/2007JCLI2044.1.

Sherwood, S. C., S. Bony, and J.-L. Dufresne, 2014: Spread in model climate sensitivity traced to atmospheric convective mixing. Nature, 505, 37-42, https://doi.org/10.1038/nature12829.

Siler, N., S. Po-Chedley, and C. S. Bretherton, 2018: Variability in modeled cloud feedback tied to differences in the climatological spatial pattern of clouds. Climate Dyn., https://doi.org/ 10.1007/s00382-017-3673-2, in press.

Soden, B. J., I. M. Held, R. Colman, K. M. Shell, J. T. Kiehl, and C. A. Shields, 2008: Quantifying climate feedbacks using radiative kernels. J. Climate, 21, 3504-3520, https://doi.org/ 10.1175/2007JCLI2110.1.
Su, H., J. H. Jiang, C. Zhai, T. J. Shen, J. D. Neelin, G. L. Stephens, and Y. L. Yung, 2014: Weakening and strengthening structures in the Hadley circulation change under global warming and implications for cloud response and climate sensitivity. J. Geophys. Res. Atmos., 119, 5787-5805, https://doi.org/ 10.1002/2014JD021642.

Tan, I., T. Storelvmo, and M. D. Zelinka, 2016: Observational constraints on mixed-phase clouds imply higher climate sensitivity. Science, 352, 224-227, https://doi.org/10.1126/ science.aad5300.

Taylor, K. E., M. Crucifix, P. Braconnot, C. D. Hewitt, C. Doutriaux, A. J. Broccoli, J. F. B. Mitchell, and M. J. Webb, 2007: Estimating shortwave radiative forcing and response in climate models. J. Climate, 20, 2530-2543, https://doi.org/ 10.1175/JCLI4143.1.

Tian, B., 2015: Spread of model climate sensitivity linked to double-intertropical convergence zone bias. Geophys. Res. Lett., 42, 4133-4141, https://doi.org/10.1002/2015GL064119.

Trenberth, K. E., and J. T. Fasullo, 2010: Simulation of present-day and twenty-first-century energy budgets of the southern oceans. J. Climate, 23, 440-454, https://doi.org/10.1175/2009JCLI3152.1.

Vial, J., J.-L. Dufresne, and S. Bony, 2013: On the interpretation of inter-model spread in CMIP5 climate sensitivity estimates. Climate Dyn., 41, 3339-3362, https://doi.org/10.1007/ s00382-013-1725-9.

Volodin, E. M., 2008: Relation between temperature sensitivity to doubled carbon dioxide and the distribution of clouds in current climate models. Izv. Atmos. Oceanic Phys., 44, 288-299, https://doi.org/10.1134/S0001433808030043.

von Storch, H., and F. W. Zwiers, 1999: Regression. Statistical Analysis in Climate Research, Cambridge University Press, 145-169.

Wood, R., and C. S. Bretherton, 2006: On the relationship between stratiform low cloud cover and lower-tropospheric stability. J. Climate, 19, 6425-6432, https://doi.org/10.1175/ JCLI3988.1.

Zhai, C., J. H. Jiang, and H. Su, 2015: Long-term cloud change imprinted in seasonal cloud variation: More evidence of high climate sensitivity. Geophys. Res. Lett., 42, 8729-8737, https:// doi.org/10.1002/2015GL065911.

Zhao, M., and Coauthors, 2016: Uncertainty in model climate sensitivity traced to representations of cumulus precipitation microphysics. J. Climate, 29, 543-560, https://doi.org/10.1175/ JCLI-D-15-0191.1.

Zhou, C., M. D. Zelinka, A. E. Dessler, and S. A. Klein, 2015: The relationship between interannual and long-term cloud feedbacks. Geophys. Res. Lett., 42, $10463-10469$, https://doi.org/ 10.1002/2015GL066698. 